\title{
NEAR EQUATORIAL ORBIT SMALL SAR CONSTELLATION FOR DEVELOPING NATIONS
}

\author{
Lawal $\mathrm{AD}^{1}$ and Radice $\mathbf{G M}^{2}$ \\ ${ }^{1}$ Researcher, ${ }^{2}$ Dr, Aerospace Engineering, University of Glasgow, Glasgow, United Kingdom, \\ a.lawal.1@research.gla.ac.uk, gianmarco.radice@glasgow.ac.uk
}

\begin{abstract}
The need to harness the benefits of space, to help urban growth and development, using cheaper space systems has become appealing to developing nations. The desired all weather radar satellite poses limitations on a small satellite. Several spaceborne Synthetic Aperture Radar (SAR) satellite configurations have been proposed to overcome the significant limit on earth surface revisit time for small SAR satellites, optimising a network of small SAR satellites dedicated to developing nations posed several challenges. In an effort to address some of these challenges, a new concept based on a constellation of small SAR satellite network, in an almost equatorial inclined orbit, operating in a multistatic configuration and solely dedicated to Equatorial region has been proposed. The network consists 3 receiver-only platforms (passive satellites) flying in formation with one transmit/receiver satellite (active satellite). Furthermore, five groundstation sites required for mission operation are located with the equatorial region. The system aims to provide 24 hours of near-real time data, over the equatorial region. A total of two orbital planes for 8 satellites are proposed. This report will describe the process of selecting a suitable orbit constellation configuration. It also determines the stability of the relative motion between the satellites within the formation in order to ensure the desired image product is consistent during operations. Furthermore, it also discusses the process of selecting suitable groundstation locations for the mission operations
\end{abstract}

Index Terms: Near Equatorial orbit, SAR, Interferometry Pendulum, constellation

\section{INTRODUCTION}

The desire to acquire near-real time, high resolution images independent of weather conditions has always been appealing to scientific and commercial industries. The need for a sophisticated system for monitoring a region such as the Equatorial Region (ER), with diverse types of valuable resources cannot be overemphasised. Within the $\mathrm{ER}^{1}$, is a host of developing nations, who stand to benefit scientifically, commercially and educationally from the implementation of an all-weather remote sensing system. Typical SAR satellites payloads are characterised by large volume, high power demand, and complexity. These, impacts on launch and mission costs, which can be unaffordable by developing nations. Recent missions have demonstrated the use of SAR payloads on smaller satellites, but there still stands a huge cost implication when a constellation of SAR satellites is considered.

The vast amount of natural resources such as animal, agricultural and human resources within the ER, can be assumed to be underutilised by harbouring nations which are mainly developing countries. In order to speed-up the development within this region, an approach entailing state-ofthe-art technology implementation is suggested. This approach requires abundance of affordable data, which can be processed for resources management and urban development. In view of the high cost implications of SAR missions, reduction of mission cost becomes paramount to ensure potential member states with ER can afford either the launch, space or ground segment costs. The concept revolves around the collaboration of several resident nations with the ER on a Multistatic SAR network mission. The geographical location of all ground segments will be within the ER region. The operation and maintenance of all mission resources will be conducted by resident nation's member of staff. The collaboration is designed to build confidence amongst each nation, in their ability to manage these resources, while enhancing development. One method of making space missions affordable is the use of small satellites to for throughput comparable to big systems.

The growing interest from the scientific community, in the application of distributed SAR systems has been ably demonstrated by the in-orbit operations of both TerraSAR-X and Tandem- $X$ in a twin satellite formation called the helix [6]. Consequently, the concept of implementing a multistatic SAR system that operates solely within the ER is considered the first step towards realising an ambition for providing affordable space mission to developing nations. In addition to acquisition of typical monostatic data products, this multistatic network will conduct interferometry operations capable for delivering a variety of data product depending on satellite configuration. 
Several spaceborne radar systems employing two or more receivers on separate platforms are commonly known as a multistatic SAR system [2]. The basic form of such a system is a Bistatic configuration, consisting of two receivers on separate platforms. The application areas of multistatic SAR configuration include but not limited to, single pass acrosstrack interferometry, single pass along-track interferometry, wide swath imaging, ground target moving indication (GTMI), spaceborne tomography, interference suppression, multistatic imaging and resolution enhancement [3]. It is evident that developing nations can immensely benefit from the availability of data provided by a multistatic SAR system, potentially serving as "spring board" for regional development.

Therefore, to design a low-cost SAR constellation mission, this paper refers to the use of a multistatic SAR constellation network in a "semi-active" configuration. The constellation is made up of transmit/receiver (monostatic SAR) satellite and several receiver-only (passive SAR) satellites orbiting around a define region. The monostatic satellite $(\mathrm{Tx} / \mathrm{Rx})$ is regarded as the master and labelled M01 and M11, while the passive satellites (Rx) are regarded as slaves and labelled S01, S02, S03 for slave plane one and S11, S12, and S13 for slave plane 2 respectively. The semi-active configuration allows the use of receiver-only satellites that whose payload can be fitted on microsatellite (typical $100 \mathrm{~kg}$ ), thereby eliminating high power demands which impact positively on satellite electric power subsystem sizing [12].

To this end, this paper aims to describe the orbit design process and the ground segment location selection process required for implementing an almost equatorial inclined LEO orbiting Multistatic SAR network

\section{ORBIT DESIGN}

The requirement for a system capable of providing 24 hours of NRT data of the equatorial region, informed the selection of an initial orbit inclination of 10 degrees. This ensures that the satellite groundtrack periodically moves between \pm 10 degrees latitude per orbit. The power demand per orbit per satellite is an area that needs to be considered. It is envisage that the technological advances in improving the conversion efficiencies of solar cells to about 40 percent [7] will help keep the satellite size at minimum since less area would be required for power generation. The satellites will be located in the LEO at an altitude of approximately $700 \mathrm{~km}$ to prevent the Van Allen belt, and ensure rapid revisit times over the region of coverage. A semi-active configuration

\subsection{Constellation}

The selected choice for achieving the ER coverage requirement considered existing satellite formations. The first formation considered was the famous cartwheel formation, a concept introduced for the SAR interferometric application with the ENVISAT mission [9]. Similar to the original concept where 3 passive satellites were proposed to image the area of the Earth illuminated by Envisat SAR, each master satellite is allocated three slave satellites for collecting returned echoes of signals transmitted by the master satellite.

A total of two orbital planes are used for the constellation: Plane one is called the "master plane" and consists of two master satellites evenly separated in time. Both master satellites share the same orbital parameters and hence have the same semi-major axis, eccentricity, inclination and (RAAN). The distance between both satellites is a result of selecting different arguments of latitude (assuming a circular orbit) for each master satellite.

Plane two is regarded as the "slave plane" which accommodates six slave satellites. Similar to the original cartwheel concept, all slave satellites share a semi-major axis, inclination and RAAN with the master satellites. However, all slave orbits are similar with slight eccentricities, with each argument of perigee symmetrically displaced (n satellites differ by $\frac{360^{\circ}}{n}$ in argument of perigee).

Originally it was proposed that initial position along the orbit selected for the master satellite should be several kilometres away in order to achieve safe distance. This configuration relies on frequently orbit maintenance manoeuvres beyond the scope of this paper. A major benefit of this configuration is the availability of varying baseline distances well suited for conducting SAR interferometry (InSAR). The elliptic relative motion experienced by the slave satellites ensures baselines are achieved in both the along-track (target velocity measurements) and across-track (target topography) interferometry. Figure 1 shows a typical cartwheel formation consisting of one master and 3 slaves. The figure is viewed from outer space over the Earth's North Pole.

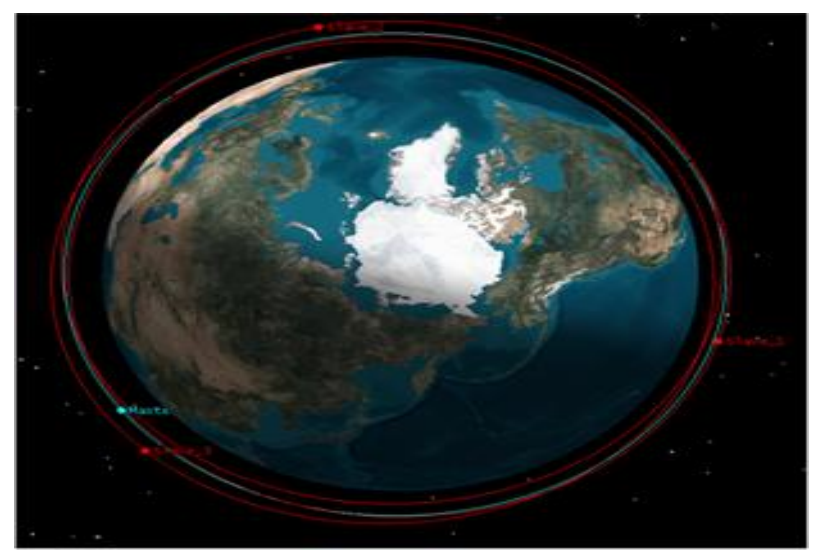

Fig -1: Typical cartwheel configuration view over the North Pole

The interferometric cartwheel formation is typified by the coupling between the vertical cross-track (radial) separation 
and the along-track displacement. An independent optimization of along-track baselines for several applications becomes impossible due to coupling between both spacecraft axis [13]. The formation hardly provides stable across-track baselines. Figure 2 illustrates a representative formation assumed for monitoring the ER as viewed from outer space through the polar axis.

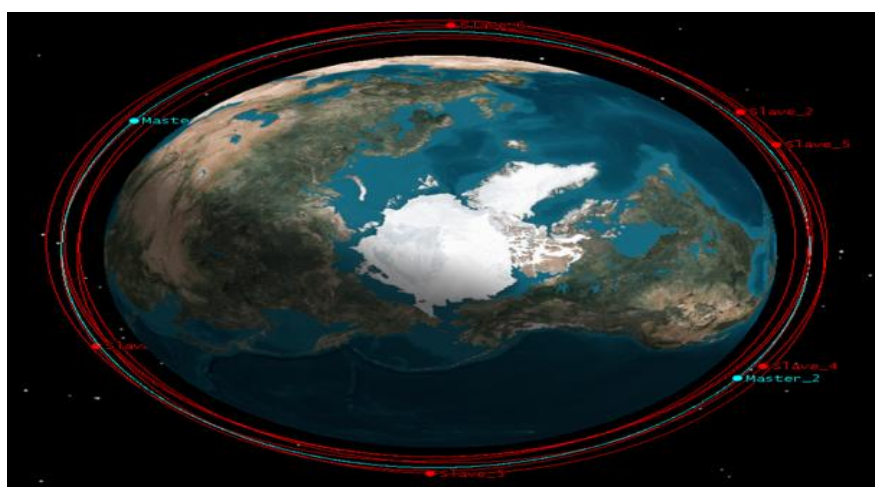

Fig -2: Orbital configuration for the SAR network using the cartwheel formation

The effects of orbit perturbation on the cartwheel formation is characterized by identical J2 secular effects, implying the ascending node and perigee precession affects all satellite equally. However, there is a secular and slow in-plane and out-plane drift experience between the master satellite and slave satellites when in cartwheel formation. The secular plane drift requires continuous orbital correction economically achievable when master satellite conducts this manoeuvre. The cartwheel formation can also be modified so that in addition to offsetting the argument of perigee of each orbit (eccentricity vectors), the various inclinations and RAAN also differ. This results in an inclined ellipse which is independent of the orbital plane of master satellite's orbit. The true angular positions of the each satellite can then be chosen as $\frac{360^{\circ}}{n}$, which yields a uniformly distributed number of slave satellites along the ellipse. This formation is characterised by constant horizontal baseline which is well suited for ground moving target indication (GMTI) and geolocation applications [5].

The second and selected formation considered, provides multiple baselines at fixed baseline ratio along the entire orbit cycle $[10,11]$. The effects of a large baseline manifests in the sensitivity to across-track interferometry, which is well suited in application areas such as digital elevation model (DEM) generation. On the other hand, short baselines are most useful for assisting in phase unwrapping, when data of undulating areas such as valleys is involved [5]. This formation is often referred to as the pendulum formation, which the original concept involved three slave satellites with the same inclination but different ascending nodes and a master satellite with a different inclination from the slave satellites. All satellites are in circular orbit and hence have equal velocities.

The concept demonstrated the use of highly inclined orbits where satellite orbits cross at the northern and southern turn. To prevent potential collision, and enhance safe operation, a slight offset to the eccentricity vector is applied. This ensures vertical separation at the point the orbits cross, with the inplane separation capable of providing cross-track interferograms at the Polar Regions [11]. The along -track baseline can always be varied depending on application, by moving the satellite along the orbit to provide GMTI or ocean current mapping [14]. Large along-track baselines can be used for measuring slow movements such as sea vessels, while short along-track baselines can be used for higher velocities such as traffic monitoring. The pendulum formation is plagued by the fact that it requires additional fuel for station keeping of the formation [5].

The major distinction between the original concept and the approach taken in this paper is that the orbits will only cross at the Equator, hence no interferograms of the polar region with be generated. The formation also differs since it consists of one master satellite dedicated to three slave satellites each. More importantly, is that two master satellites share the same orbital plane, but are separated in time (orbital period/2). Similarly, the slave satellites also share the same orbital plane and inclination but different RAAN.

One set of slave satellites (three) are dedicated to one master satellite and the other set are separated in time (orbital period/2) from the first set. The slave satellites are ahead of the master satellites for safe operation, and in event that the orbits cross at the equator, the altitude of the slave satellite would be lower than the master satellite due to different ballistic coefficients. Figure 3 and 4 illustrate 2D and 3D the application of the pendulum configuration for a near equatorial orbit using Satellite Tool Kit (STK) software.

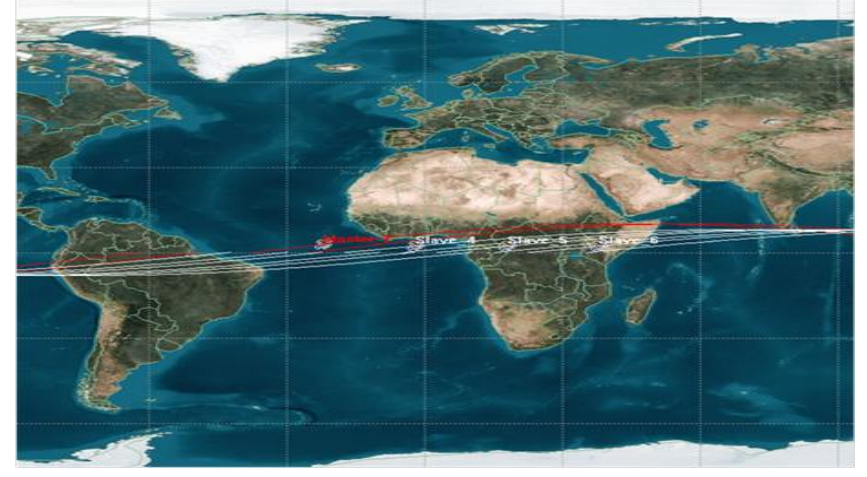

Fig -3: STK 2D representation of Pendulum formation over the Equatorial Region

It is pertinent to note that there are other configurations such as the Carpe not discussed in this paper. The Carpe is a 
combination of the cartwheel and pendulum configuration where the satellites have the same inclination and different RAAN. A thorough evaluation of the carpe formation and will be evaluated in the near future.

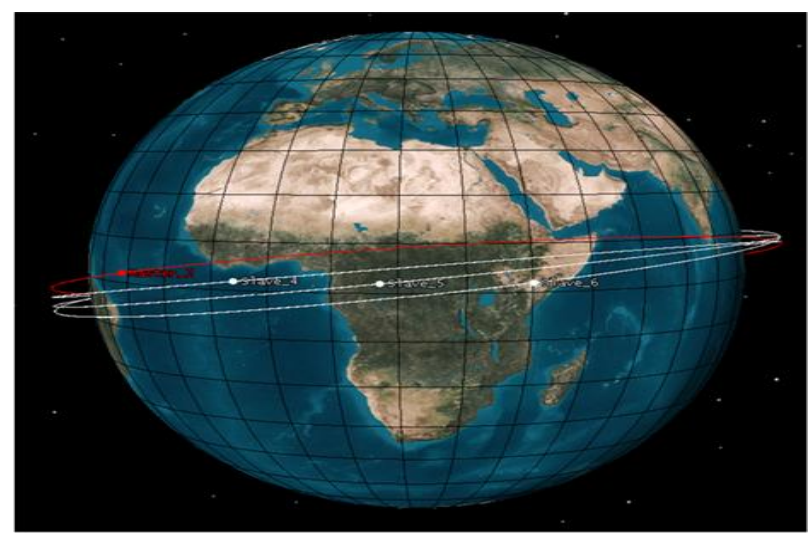

Fig -4: STK 3D Representation of Pendulum formation over the Equatorial Region

\subsection{Constellation Analysis}

To conduct an initial analysis of the constellation design, the concept of formation flying is explored in order to get an insight of possible optimization techniques that can be implemented. The use of multiple satellites flying in formation with a specific geometry prevents the substantial financial and technical challenges of building a radar dish of equivalent size [16]. Although formation flying involves two concepts: one involving two or more spacecraft docking; and spacecraft flying in formation with the aim of maintaining relative positions. The latter is the concept which is explored in this work.

The spacecraft formation considered involves several satellites of equal type and build. This ensures that each satellite ideally has equal ballistic coefficient and therefore experience orbit decay at a similar rate $[17,18]$. Thus this assumption allows the possibility of analytically finding closed relative orbits [16]. The close relative orbit as seen by the spacecraft rotating reference frame, describes a geometry that can either be circular or elliptic. Figure 4 shows the definition of the reference frame for satellites in close formation using just two satellites (One master or reference satellite and one slave). In various text reference materials $[17,18,19]$, the satellites are often referred to as "Chief and Deputy" respectively. The formation illustrates a general type with out-of-plane relative motion between the satellites. This reference frame is often called the Hill reference frame (o frame), who's origin is at the master satellite position with vectors mathematically expressed as

$$
\begin{gathered}
\widehat{\boldsymbol{o}_{r}}=\frac{\boldsymbol{r}_{c}}{r_{c}} \\
\widehat{\boldsymbol{o}_{\theta}}=\widehat{\boldsymbol{o}_{h}} \times \widehat{\boldsymbol{o}_{r}}
\end{gathered}
$$

$$
\widehat{\boldsymbol{o}_{h}}=\frac{\boldsymbol{h}}{h}
$$

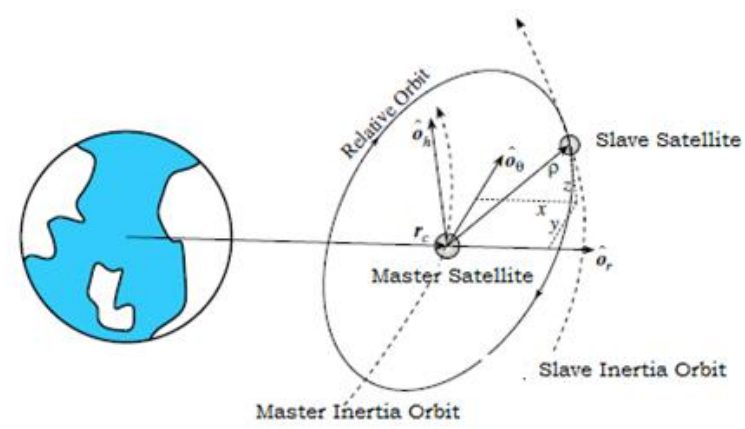

Fig -5: Hill coordinates reference frame definition for satellite formation [19]

Where the master satellite inertia position is expressed by the vector $\boldsymbol{r}_{c}(t)$ and the slave position expressed by vector $\boldsymbol{r}_{d}(t)$.The Master satellite is the satellite about which all other (slave) satellites fly in formation and ideally appear to orbit around it. The o frame $\widehat{\boldsymbol{o}_{\boldsymbol{r}}}$ is in the master satellite orbit radius direction, ${ }^{\boldsymbol{o}_{\boldsymbol{h}}}$ is parallel to the orbit momentum vector (i.e vector cross product of master satellite's position and velocity vectors), and $\boldsymbol{o}_{\boldsymbol{\theta}}$ completes the traid. Therefore the relative orbit position vector $\rho$ is expressed in the o frame as $\rho=(x, y, z)^{T}$

By satisfying some constraints such as assuming a circular orbit, the relative orbit can be bounded to ensure that the spacecraft do not drift apart [5, 16, 17], thus leading to the simplest form of Clohessy-Wiltshire Equations below.

$$
\begin{gathered}
\ddot{x}-2 n \dot{y}-3 n^{2} x=0 \\
\ddot{y}+2 n \dot{x}=0 \\
\ddot{z}+n^{2} z=0
\end{gathered}
$$

Reference [16] provides the derivation of the relationship between relative positions and orbital elements and the constants for mapping the orbital elements to $\mathrm{x}, \mathrm{y}, \mathrm{z}$ coordinate. The resulting equations are

$$
\begin{gathered}
x(t)=A_{0} \cos (n t+\alpha) \\
y(t)=-2 A_{0}(n t+\alpha)+\Delta y \\
z(t)=B_{0} \cos (n t+\beta)
\end{gathered}
$$


Where A0, B0, $\alpha$ and $\beta$ are given by the differences in the inclination, argument of perigee, and mean anomaly given by;

$$
\begin{gathered}
A_{0}=-a * \Delta a \\
B_{0}=a * \sqrt{\left(\Delta i^{2}+(\sin (i))^{2} * \Delta^{2}\right)} \\
\alpha=0 \\
\theta=\operatorname{atan}\left(\frac{\Delta i}{-\sin (i) * \Delta \Omega}\right. \\
\beta=\omega-\theta \\
\Delta y=0
\end{gathered}
$$

Using Clohessy-Wiltshire equation, a MATLAB program was run to calculate the relative position of the slave satellites while in a pendulum configuration. This was conducted by using the master satellite and one slave satellite. The initial orbital elements which had been earlier derived based on user requirements and applications served as input into the program. Orbital element values for master satellite are as follows:

- Semi-major axis (a) km: 7078.14

- Eccentricity $(e): 0$

- Inclination $(i)$ deg : 10

- $\quad \operatorname{RAAN}(\Omega) \operatorname{deg}: 0$

- Argument of Perigee $(\omega)$ deg : 0

- Mean Anomaly $(M) \operatorname{deg}: 0$

Orbital element differences are as follows:

- $\Delta a: 0$

- $\Delta e: 0$

- $\Delta i: 2$

- $\Delta \Omega: 20$

- $\Delta \omega: 0$

- $\Delta M: 5$

Therefore slave satellite initial orbital elements were calculated as by adding the orbital element differences and mapping into the $\mathrm{x}, \mathrm{y}, \mathrm{z}$ coordinate system as described above. Figure 6 is a plot of the slave relative position over 24 hours, which shows that the relative motion is simply oscillatory in the out-of -plane direction due to difference in inclination angle. A maximum across-track separation of $500 \mathrm{~km}$ is achievable. The in-plane distance for both $\mathrm{x}$ and $\mathrm{y}$ directions remain constant as expected for a typical pendulum configuration. It is duly noted that other assumptions include the fact that Clohessy-Wiltshire equation is based on the twobody equation and therefore the effects of perturbations is not considered.

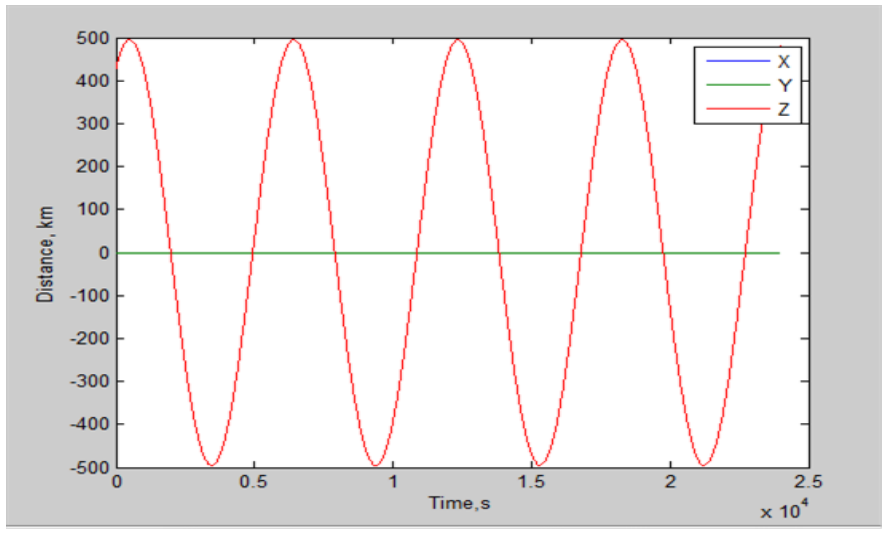

Fig -6: STK 3D Representation of Pendulum formation over the Equatorial Region

\section{GROUND SEGEMENT DESIGN}

The desire to locate all mission resources input and output within the ER implies all ground segment are geographically located between latitudes \pm 10 degrees of the Equator. The selection of ground station locations commenced by populating every country whose border lies within the ER and in some cases, Islands were also considered. Table 1 is an

\begin{tabular}{|c|c|c|c|c|}
\hline Latitude $\Psi$ & Longitude - & City, State & $\nabla$ & Country \\
\hline $3^{\circ} 35^{\mathrm{N}} \mathrm{N}$ & $98^{\circ} 40^{\prime} \mathrm{E}$ & Medan & & ....Jonesia \\
\hline $9^{\circ} 59 \mathrm{~N}$ & $83^{\circ} 02 \mathrm{~W}$ & Limon & & "्रsta Rica \\
\hline $8^{2} 07 \mathrm{~N}$ & $100^{\circ} 22 \mathrm{E}$ & nor Star, Kedah & & IVialaysia \\
\hline $4^{\circ} 53 \mathrm{~N}$ & $114^{\circ} 58 \mathrm{E}$ & Bandar Seri Begawan & & orunei \\
\hline $8^{2} 14 \mathrm{~N}$ & $75^{\circ} 34^{\prime} \mathrm{W}$ & Medellín & & lombia \\
\hline $8^{\circ} 30^{\prime} \mathrm{N}$ & $2^{\circ} 38 \mathrm{E}$ & Porto-Novo & & nin \\
\hline $8^{\circ} 58^{\mathrm{N}}$ & $79^{\circ} 32 \mathrm{~W}$ & Panama City & & Shama \\
\hline $6^{2} 49 \mathrm{~N}$ & $5^{\circ} 17 \mathrm{~W}$ & Yamoussoukro & & Dte d'Ivoire \\
\hline $9^{\circ} 30^{\prime} \mathrm{N}$ & $44^{\circ} 0 \mathrm{E}$ & Feis s, Somaliland & & *malia \\
\hline $3^{\circ} 25^{\prime} \mathrm{N}$ & $78^{\circ} 32 \mathrm{~W}$ & Santiago de Cali & & lombia \\
\hline $7^{\circ} 45 \mathrm{~N}$ & $8^{2} 49 \mathrm{~W}$ & Nzérékoré & & Ifinea \\
\hline $9^{2} 04 \mathrm{~N}$ & $7^{\circ} 29 \mathrm{E}$ & Abuja & & Nweria \\
\hline $4^{\circ} 22^{\prime} \mathrm{N}$ & $18^{\circ} 35^{\prime} \mathrm{E}$ & Bangui & & Entral African Republic \\
\hline $4^{\circ} 55^{\mathrm{N}} \mathrm{N}$ & $1^{\circ} 46 \mathrm{~W}$ & Sekondi-Takoradi & & Gana \\
\hline $9^{\circ} 24 \mathrm{~N}$ & $0^{\circ} 51 \mathrm{~W}$ & Tamale & & Eivana \\
\hline $8^{\circ} 48 \mathrm{~N}$ & $58^{\circ} 10^{\circ} \mathrm{W}$ & Georgetown & & Suyana \\
\hline $5^{2} 31^{\prime} \mathrm{N}$ & $95^{\circ} 25^{\prime} \mathrm{E}$ & Banda Aceh & & .... Jonesia \\
\hline $8^{2} 08 \mathrm{~N}$ & $1012 \mathrm{E}$ & Lomé & & =go \\
\hline $4^{\circ} 03^{\prime} \mathrm{N}$ & $9^{\circ} 41 \mathrm{E}$ & Douala & & meroon \\
\hline $5^{\circ} 20^{\prime} \mathrm{N}$ & $4^{\circ} 01 \mathrm{~W}$ & Abidjan & & Llte d'lvoire \\
\hline $9: 30^{\prime} \mathrm{N}$ & $13^{\circ} 42 \mathrm{~W}$ & Conakry & & Ilinea \\
\hline $9^{\circ} 02^{2} \mathrm{~N}$ & $38^{\circ} 44^{\prime} \mathrm{E}$ & Addis Ababa & & ethiopia \\
\hline $7^{\circ} 53^{\mathrm{N}} \mathrm{N}$ & $72^{\circ} 30^{\prime} \mathrm{W}$ & Cúcuta & & ombia \\
\hline $5^{2} 52 \mathrm{~N}$ & $55^{\circ} 10^{\circ} \mathrm{W}$ & Paramaribo & & - riname \\
\hline $4^{\circ} 37^{\prime} \mathrm{N}$ & $74^{\circ} 05^{\mathrm{W}}$ & Bogotá & & ombia \\
\hline $8^{\circ} 55 \mathrm{~N}$ & $158^{\circ} 11 \mathrm{E}$ & Palikir & & iederated States of Micronesia \\
\hline $8^{\circ} 34^{\prime} \mathrm{N}$ & $18^{2} 05 \mathrm{~W}$ & Moundou & & Inad \\
\hline $4^{\circ} 45 \mathrm{~N}$ & $7^{\circ} 0^{\prime} \mathrm{E}$ & Port Harcourt & & Averia \\
\hline $9^{\circ} 58^{\prime} \mathrm{N}$ & $84^{\circ} 05^{\mathrm{W}}$ & San José & & ए्णsta Rica \\
\hline $8^{\circ} 30^{\prime} \mathrm{N}$ & $13^{2} 7 \mathrm{~W}$ & Freetown & & rra Le one \\
\hline
\end{tabular}
extract of the geographical locations of countries whose boundaries lie within the ER. A total number of 49 countries/Islands have boundaries within the ER.

Table -1: Countries and Islands whose geographical boundary lie with the ER 
The choice of groundstation location was based on several criteria:

- Level of involvement in space technology

- Location of boundary within the ER

- Potential ability to afford space missions

Although several other criteria are still being evaluated, the ones listed were required to provide baseline idea of selecting the groundstation locations. An initial number of 12 ground station sites were selected on the basis of ensuring overlap between successive coverage ranges using a minimum elevation angle of 6 degrees.

However, due to possibility of interference and need for reduction in mission cost, the ground station locations were reduced to 5. An access report from a simulation run over 24 hours using STK shows that there were varying access times for each groundstation. A maximum of 14 passes and minimum of 13 passes was experienced by each ground station per satellite. An overall mean duration of 149 minutes is available to each satellite for downlinking data to each groundstation. This implies a total of 745 minutes available each satellite in the SAR network for downlinking data to groundstation in view.

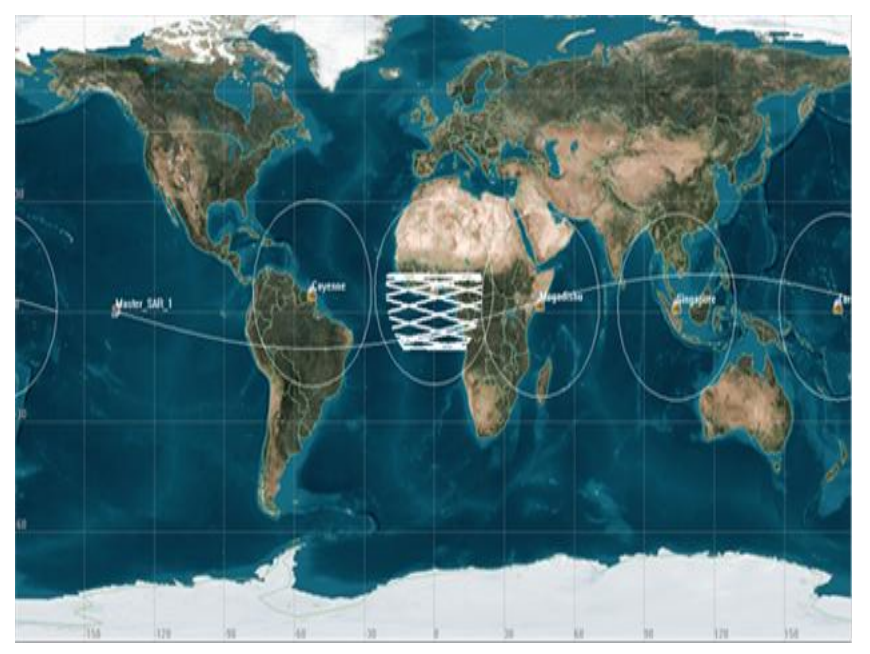

Fig -7: STK 2D visualisation of groundstation locations masks

The groundstation locations can be varied with little or no change in the access times, depending on interested nations; however, an optimum configuration would require a maximum of 5 groundstation sites. Operational strategies that outline communication protocol and priority/ conflict management must be defined. Data transfer methods from archiving units of one site to the other must also be defined.

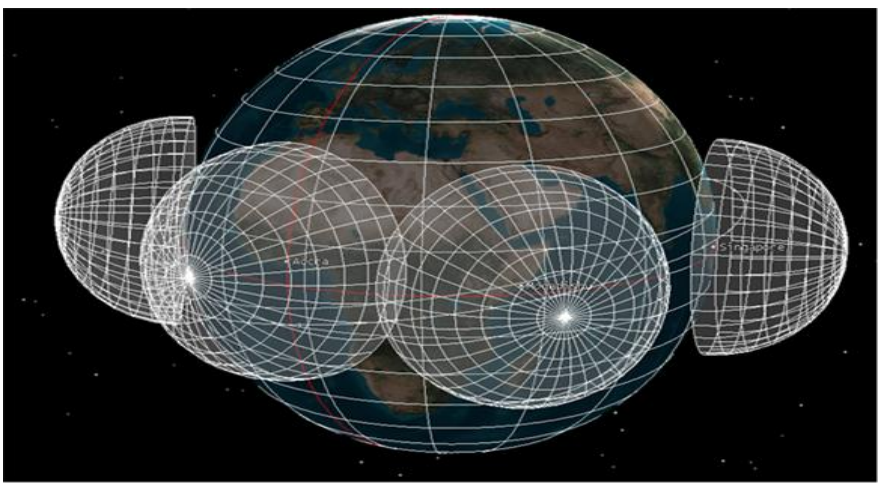

Fig -8: STK 3D visualisation of ground station location masks

\subsection{Access to selected ground segment sites}

As mentioned in section 1 , there are over 45 countries within the ER, with only 5 selected as possible locations for ground segment sites. These locations are subject to flexibility although certain criteria such as having existing space agency, and proximity to Equator were considered.

Fig shows a Northerly oriented view of the locations of the selected ground stations.

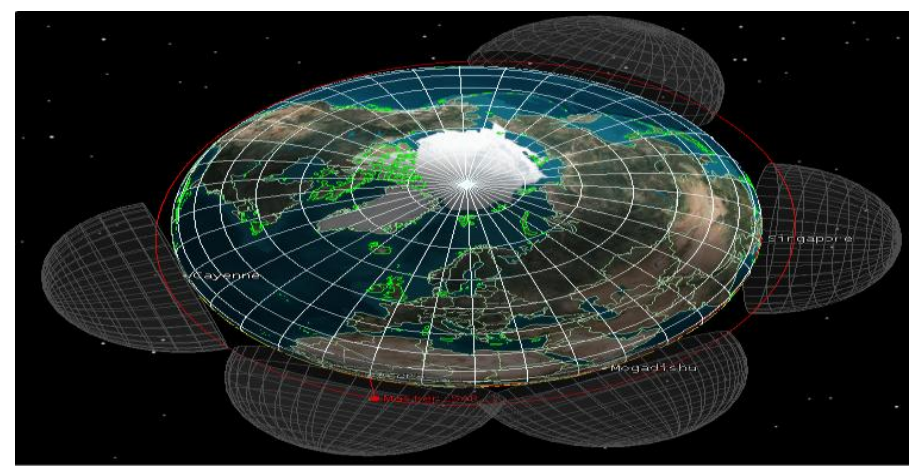

Fig 9: Selected locations for Ground Segment - View from the North Pole

The distance between subsequent locations varies; however, the maximum time between successive contacts with groundstation must be less than 10 minutes. Table 1 details the location of the selected ground station sites. Sites can be flexibly selected provided the out of communication time with satellites is less than 10 minutes.

Table 2: Location of selected ground segment sites with ER

\begin{tabular}{|l|l|l|}
\hline Country & Latitude & Longitude \\
\hline Accra & $5^{\circ} 30^{\prime} \mathrm{N}$ & $0^{\circ} 10^{\prime} \mathrm{W}$ \\
\hline Cayenne & $4^{\circ} 56^{\prime} \mathrm{N}$ & $52^{\circ} 20^{\prime} \mathrm{W}$ \\
\hline Mogadishu & $2^{\circ} 04^{\prime} \mathrm{N}$ & $45^{\circ} 22^{\prime} \mathrm{E}$ \\
\hline Singapore & $1^{\circ} 17^{\prime} \mathrm{N}$ & $103^{\circ} 51^{\prime} \mathrm{E}$ \\
\hline Tarawa & $1^{\circ} 19^{\prime} \mathrm{N}$ & $172^{\circ} 58^{\prime} \mathrm{E}$ \\
\hline
\end{tabular}




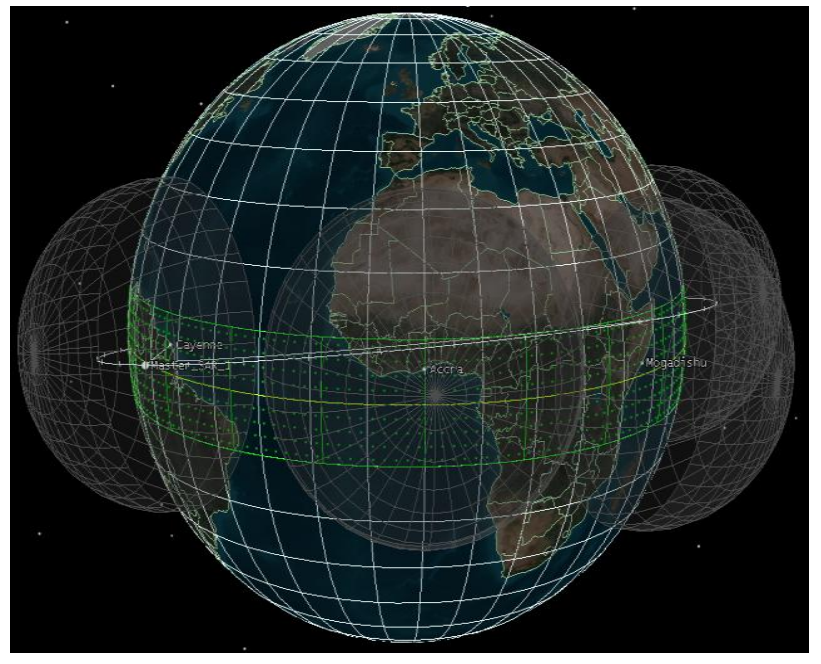

Fig10: Ground segment location showing M01 making contact with groundstation in Accra

The access to the selected ground segment location allows each group of the satellite constellation to always be in view of one ground station.

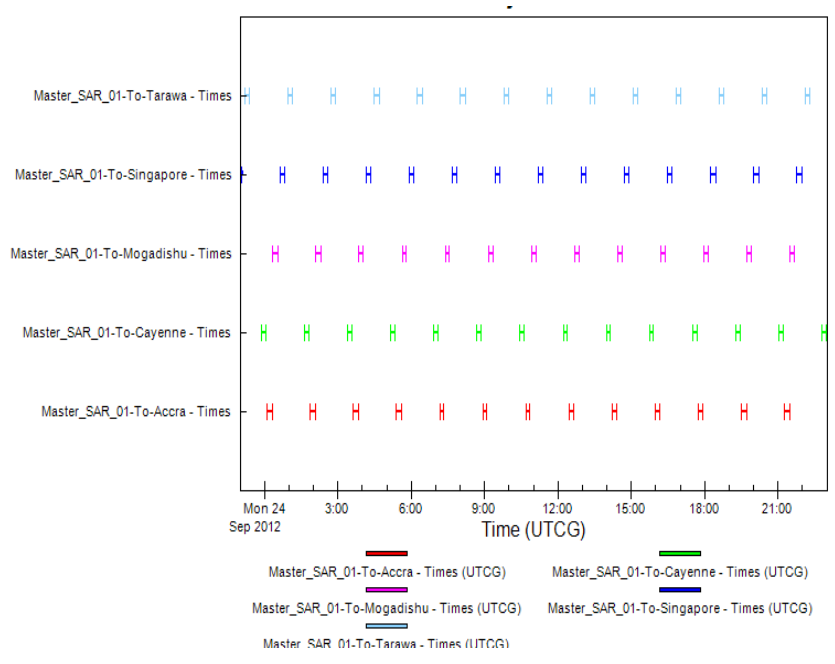

Fig 11: Access report of M01 to selected ground sites within ER

Fig summarizes a daily access report for M01 to each ground segment. A total of 70 accesses daily are available to download captured data, or upload telecommand to each spacecraft as required. This configuration serves to usher a new approach in data generation.

Using a line of sight constraint of a minimum of 6 degrees elevation angle is applied to each groundstation. Therefore, the available downlink time translates to approximately half of a sidereal day. Figure 11 is a 24 hours extract of the access report in STK for M01 to Cayenne and Mogadishu.

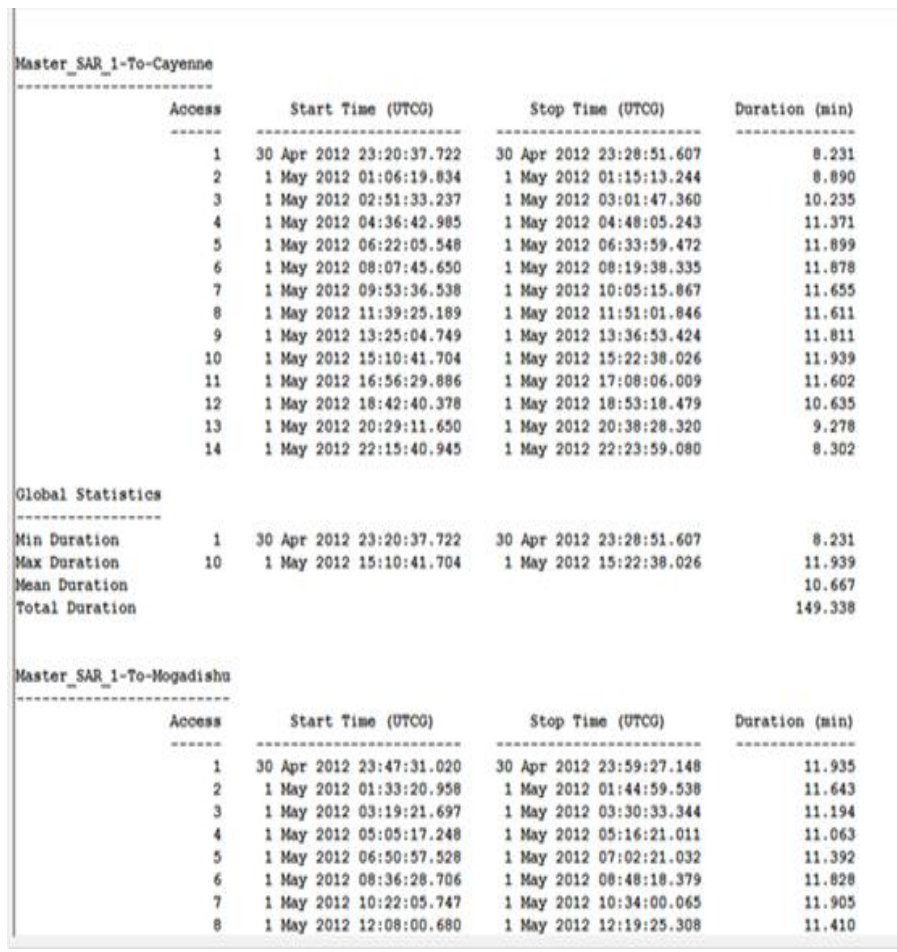

Fig -12: Extract of access report for M01 to Cayenne and Mogadishu over 24 hours duration

\section{CONCLUSIONS}

This paper identifies the need for a dedicated all weather SAR system to provide continuous high quality data products to developing nation. Developing nation form majority of the countries with the equatorial region and therefore, the study proposes the establishment of a consortium of developing nations with the equatorial region solely for the realisation of a network of small SAR satellites, whose resources are sole dedicated to the equatorial region. The thrust of this work is to provide a cheap and affordable solution to space mission for developing nations.

The approach taken is a semi-active configuration consisting of receiver only satellites in formation with traditional monostatic SAR satellites for the acquisition of various data products. The constellation consisting of mainly small satellites is capable of functioning as a larger system. Two main distributed SAR configurations were considered, and the pendulum configuration was preferred to the cartwheel mainly due to the variety of application areas it offers, at the expense of additional fuel for station keeping. The pendulum formation has never been adopted for a near equatorial LEO orbit and therefore presents new challenges that require further investigations.

The SAR network comprises of both space and ground segments, with the later consisting of 5 potential groundstation sites located at different geographical location within the ER. 
Preliminary results show that a near 24 hours data availability is feasible with all small satellites fully operational and serving as a "Big system". However, resource management procedures need to be defined in order to avoid conflict.

The approach suggests the formation of a consortium of developing nations within the ER that could exploit advantages such as overall reduction in cost of space mission due to shared cost, availability of cheaper data, availability of a variety of data products, and opportunity to apply the data for various form of development.

Five sites were selected as locations for ground segments, which will facilitate a nearly 24 hours availability of data with a total of 70 access passes to the ground segments.

\section{ACKNOWLEDGEMENTS}

I would like to extend my sincere appreciation to my kids, Moesha and Leo Lawal. The thought of ensuring you both live in a better world spurred me on in my journey to realize new concepts for developing nations. My undying gratitude to Dr, Gianmarco Radice, for you kindness, patience and support all through my time with you as a research student. To my Dad, Abdul Lawal, your spirit never left my side as it guided me all the way even through hard times. Finally, to all the fellow researchers, friends and colleagues who contributed to this work, I will forever be grateful.

\section{REFERENCES:}

[1] Krieger, G and Moreira, A., Spaceborne Interferometric and Multistatic SAR Systems

[2] Chernyak, V.S. (1998) Fundamentals of Multisite Radar Systems, Gordon and Breach, Amsterdam.

[3] Krieger, G. and Moreira, A. (2006) Spaceborne bi- and multistatic SAR: potential and challenges, IEE Proc. Radar, Sonar and Navigation, 153 (3), 184-98.

[4] Krieger, G., Papathanassiou, K., Cloude, S., Moreira, A., Fiedler, H. and Volker, M. (2005) Spaceborne polarimetric SAR interferometry: performance analysis and mission concepts, in 2nd ESA International Workshop on Applications of Polarimetry and Polarimetric Interferometry (PolInSAR), Frascati, Italy.

[5] Torres, R., Lokas, S., Moller, H.L., Zink, M. and Simpson, D.M. (2004) The TerraSAR-L mission and system, in Proceedings of the IEEE International Geoscience and Remote Sensing Symposium (IGARSS'04), Anchorage, Alaska.

[6] Cherniakov, M. (2008) Bistatic Radar: Emerging Technology. Wiley \& Son, Ltd

[7] Giese, C., (2011) TANDEM-X, Presentation at the Science Team Meeting, Astrium

[8] Martin, A et al., (2012). Solar Cell Efficiency Tables version 39. Progress in Photovoltaics: Research and Application. Vol 20. Issue 1. pp12-20.

[9] Raney, R. K., Porter, D. L. and Monaldo, F. M. (2002) Bistatic WITTEX: an innovative constellation of radar altimeter satellites, in Proceedings of the International Geoscience and Remote Sensing Symposium (IGARSS,02), vol. 3, pp. 1355-7.

[10] Massonnet, D. (2001) Capabilities and limitations of the interferometric cartwheel, IEEE Trans., GRS-39 (3), 506-20.

[11] Zink, M., Krieger, G. and Amiot, T. (2003) Interferometric performance of a cartwheel constellation for TerraSAR-L, ESA Fringe Workshop, Frascati, Italy.

[12] Fiedler, H. and Krieger, G. (2004) Close formation of micro-satellites for SAR interferometry, 2nd International Symposium on Formation Flying, Washington, NASA GSFC.

[13] Wanwiwake, T., Underwood, C. (2010) A Bi/Multi-Static Microsatellite SAR Constellation, Small Satellite Missions for Earth Observation, DOI 10.1007/798-3-642-03501-2, (C) Springer-Verlag Berlin Heidelberg 2010

[14] Das, A. and Cobb, R. (1998) TechSat 21 - space missions using collaborating constellations of satellites, in 12th AIAA/USU Conference on Small Satellites, Utah.

[15] Bethke, K.H., Baumgartner, S., Gabele, M., Hounam, D., Kemptner, E., Klement, D., Krieger, G. and Erxleben, R. (2006) Air- and spaceborne monitoring of road traffic using SAR/MTI project TRAMRAD, ISPRS J. Photogrammetry and Remote Sensing, 61 (3-4), 243-259.

[16] H. Schaub and J. Lunkins. Analytical Mechanics of Space Systems. AIAA Educations Series, 2003

[17] Vallado, A.D. and McClain, W.D. Fundamentals of Astrodynamics and Applications. 3rd ed. 2007. Microsm Press and Springer. New York. ISBN 978-1-881883-14-2

[18] Wertz, J. R. and Larson, W.J., Space Mission Analysis and Design. 3rd ed. 2007. Microsm Press and Springer. New York. ISBN 978-1881883-10-4.

[19] H. Schaub (2002). Spacecraft Relative Orbit Geometry Description through Orbit Element Differences. 14th U.S. National Congress of Theoretical and Applied Mechanics Blacksburg, VA, June 23-28

\section{BIOGRAPHIES:}

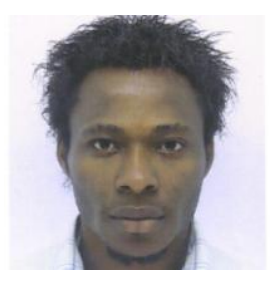

Abdul Duane Lawal is currently a research student of the Aerospace department of the university of Glasgow. His previous experiences include, the system engineer of NigeriaSat-X mission at SSTL. He is currently researching on distributed SAR systems.

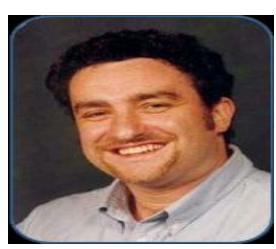

Gianmarco Radice is a Reader at the University of Glasgow. He is currently the Director of space Programmes at University of Glasgow Singapore, Singapore Polytechnic Campus. He's research interest include: Space Mission Analysis and Design, Orbit Mechanic and Astrodynamics. 